\title{
Autoren des Heftes
}

Dr. Heinz Bolsenkötter, WIBERA Wirtschaftsberatung AG, ute.leidig@de.pwc.com

Prof. em. Dr. Dr. h. c. mult. Peter Eichhorn, Universität Mannheim, Lehrstuhl für Allgemeine Betriebswirtschaftslehre, Public \& Nonprofit Management, peter.eichhorn@uni-mannheim.de

Lukas Haag, IEW, Universität Zürich, lhaag@iew.unizh.ch

Prof. Dr. Ashok Kaul, Johannes Gutenberg-Universität Mainz, FB 03 - Recht und Wirtschaft, ashok.kaul@uni-mainz.de

Prof. Dr. Dirk Meyer, Helmut-Schmidt-Universität, Universität der Bundeswehr Hamburg, Institut für Wirtschaftspolitik, dirk.meyer@hsu-hh.de

Ass.-Prof. Dr. Guido Offermanns, Alpen-Adria-Universität Klagenfurt, Österreich, Fakultät für Wirtschaftswissenschaften, Abteilung für Öffentliche Betriebswirtschaftslehre, guido.offermanns@uni-klu.ac.at

Prof. Dr. Hans Jürgen Rösner, Universität zu Köln, Seminar für Genossenschaftswesen, roesner@wiso.uni-koeln.de

Prof. Dr. Michael Ronellenfitsch, Eberhard-Karls-Universität Tübingen, Lehrstuhl für öffentliches Recht, michael.ronellenfitsch@jura.uni-tuebingen.de

Dr. Georg von Schnurbein, Universität Basel, Schweiz, Kompetenzzentrum für Stiftungswesen und Philanthropie, georg.vonschnurbein@unibas.ch

Prof. Dr. Frank Schulz-Nieswandt, Universität zu Köln, Seminar für Sozialpolitik, schulz-nieswandt@wiso.uni-koeln.de 